\title{
From scientific evidence to media and policy: Wine - part of a balanced diet or a health risk?
}

\author{
U. Fradera ${ }^{1,2, a}$ and C. Stein-Hammer ${ }^{1}$ \\ ${ }^{1}$ Deutsche Weinakademie, Bodenheim, Germany \\ ${ }^{2}$ Coordinator, Wine Information Council (WIC)
}

\begin{abstract}
Recently, some studies [1,2] and media reports alerted scientists, health and wine professionals alike. The health benefits of moderate wine consumption were questioned with headlines such as "One drink a day can shorten life" and "Moderate drinking guidelines are too loose, study says". One publication [2] asserted that there is no safe level of consumption and that the adverse health effects associated with the consumption of alcoholic beverages, including wine, outweigh any beneficial effects; this kind of media attention not only contributed to a considerable uncertainty among moderate wine drinkers but also among physicians advising their patients. What about the existing body of evidence and the research results during the last two and half decades that have established the J-curve? It describes the association between the consumption of alcoholic beverages and the total mortality where light to moderate drinkers are at a lower risk of death from all causes than either abstainers or heavy drinkers. However, excessive drinking is always associated with an increased risk of death and various health risks. Are these previous scientific data no longer valid? Is cutting down or even eliminating wine consumption a public health goal? A detailed scientific analysis of these publications will be provided and discussed whether the drinking guidelines worldwide should be adjusted. The risk of moderate wine drinkers within the scope of other risk factors will be examined.
\end{abstract}

During the last year, some studies [1,2] and media reports alerted scientists, health and wine professionals alike. The health benefits of moderate wine consumption were questioned with headlines such as "One drink a day can shorten life" and "Moderate drinking guidelines are too loose, study says". One publication [2] asserted that there is no safe level of consumption and that the adverse health effects associated with the intake of alcoholic beverages, including wine, outweigh any beneficial effects. This kind of media attention not only contributed to a considerable uncertainty among moderate wine drinkers but also among physicians advising their patients.

\section{Global Burden of Disease Collaborator Study}

The Global Burden of Disease Collaborator (GBD) consortium carried out a large meta-analysis [2], merging data from 694 observational studies with a combined coverage of 28 million people in 195 countries, to estimate the levels of alcohol consumption worldwide and the associated health risks. The researchers pooled alcohol sales data with the prevalence of alcohol drinking and abstinence, self reported data on the amount alcohol consumed and tourism data, estimating levels of illicit trade and home brewing and then merged all this information into mathematical models to predict the harm from alcohol worldwide. Then the effects of alcohol consumption on a list of 23 alcohol-related health outcomes were examined.

a e-mail: fradera@deutscheweinakademie.de
They found that pooling the 23 health outcomes indicated a $0.5 \%$ increase in relative risk for moderate drinkers, defined as persons consuming up to one standard drink every day when compared with abstainers $(7 \%$ for two drinks and $37 \%$ for five drinks per day, respectively) and concluded that the protective effect of alcohol for some health conditions was offset by the risks for others and that there is no safe level of alcohol intake. Based on these results, the authors recommended to revisit worldwide alcohol control policies and health programmes and consider recommendations for abstaining from alcohol.

These findings seem to imply that even one glass of wine per day carries a health risk but what does it mean in practical terms?

\section{Study limitations}

To evaluate the practical implications, a careful analysis of the publication showed:

- The GBD study is a population-level study but the results are being interpreted at an individual level. 23 alcohol-related health issues were merged together, however, not everyone experiences them at the same rate. For example, for diabetes and heart disease, the risk decreases with light and moderate drinking. According to the authors, this benefit is neutralized by the increased risk for cancer and tubercolosis. Yet for many individuals, diabetes and heart disease pose a much higher risk than cancer and tuberculosis.

- Many lifestyle [3] and cultural factors [4] strongly modify the association between the consumption of 
alcoholic beverages and disease outcomes [5]. Such modifying factors include the pattern of drinking (regular moderate versus binge drinking) [6], the type of beverage (wine versus beer and spirits) [7,8], drinking with or without a meal $[9,10]$, the socioeconomic status of the individual [11], lifestyle factors such as diet [12], physical activity [13], etc. Not taking into account such factors, the estimated intake of a given amount of alcohol provides an incomplete assessment of the effects of alcohol on health.

- The authors focus in their conclusion that their analyses have implications for setting alcohol policy around the world and on ways of decreasing the intake of alcoholic beverages through changes in drinking guidelines. However, their lack of considering specifics of the culture/population for which the policy is being suggested, such as evaluating lifestyle factors, the pattern of drinking, cultural factors, underreporting of alcohol intake [14], etc., negates the ability to provide useful information applicable to a single population. Their statement that zero intake of alcohol would be preferable everywhere is contradicted by consistent data from numerous large cohort-studies which indicate that non-drinkers have higher risks of cardiovascular disease and total mortality than regular moderate drinkers who do not binge drink [15].

The overall combined results from such divergent populations as in the GBD study have little applicability in setting guidelines that would prevent alcohol abuse in any specific group of individuals around the world - they apply to no specific population. Thus, it is important to carefully interpret and analyse population-based data and their respective conclusions. This study does not suggest that drinking moderately will increase an individual's risk of disease or death - it only presents correlations on a population level. In addition, the association shown here was not causal!

This study confirms, however, that excessive drinking carries many health risks.

\section{Communication versus education}

The objective of drinking guidelines as well as public health campaigns/policy is to change the behaviour of individuals to consume alcoholic beverages responsibly. Such behaviour change is only possible with education, so consumers can make informed decisions. Consequently, study findings need to be translated into measures/messages that make sense to individuals.

In an attempt to communicate that moderate drinking is an important public health risk for women and to draw the public's attention to the fact that even moderate levels, e.g. one bottle of wine per week, can put individuals at risk of cancer, particularly breast cancer (the most commonly occuring cancer in women in the UK), the alcohol-related cancer risk was calculated in terms of cigarette equivalents [16]. The authors hope by using cigarettes as comparator, they could communicate this message in a more impactful way.

The researchers have estimated the risk of cancer associated with drinking moderate levels of alcoholic beverages and compared this to the risk of smoking. They calculated that in non-smoking men, the absolute lifetime risk of cancer associated with drinking one bottle of wine per week is $1 \%$. For women, it is $1.4 \%$. This means, if 1000 men and 1000 women each drank one bottle of wine per week, around 10 extra men and 14 extra women may develop cancer at some point in their life. This is the equivalent to smoking roughly five cigarettes per week for men and ten cigarettes per week for women. Drinking three bottles of wine was associated with an increase of absolute lifetime risk to $1.9 \%$ in men and $3.6 \%$ in women, or 19 in 1000 men and 36 in 1000 women, respectively. In men, this risk appears to be associated primarily with cancers of the gastrointestinal tract, whereas in women, the risk seems to be associated with breast cancer.

The authors conclude: Our estimation of a cigarette equivalent for alcohol provides a useful measure for communicating possible cancer risks that exploits successful historical messaging on smoking. It is well established that heavy drinking is linked to cancer of the mouth, throat, bowel, liver and breast. Yet, in contrast to smoking, this is not widely understood by the public. By using cigarettes as comparator, this message could be communicated more effectively to help individuals make more informed lifestyle choices [16].

How useful is this method to communicate the possible risks associated with excessive drinking to respective target groups? Can moderate wine consumption still be part of balanced diet when considering the results of this study?

\section{Wine a part of a balanced diet or a health risk?}

After a thorough analysis, the following points should be considered:

1. The authors explain: This study is not saying that drinking alcohol is in any way equivalent to smoking.

2. The alcoholic beverage that is mentioned as comparator is wine, however, only alcohol consumption was estimated in the study and not wine per se.

3. The findings have been generated through a mathematical model and are not based on a prospective cohort study following up participants of a particular population for a certain period of time. The relative risk due to cigarette smoking is based on certain assumptions and the risks for the very low levels of smoking were extrapolated from higher levels of smoking using a statistical model.

4. Data are limited to alcohol and/or tobacco-related cancers. While both moderate intake of alcoholic beverages and a few cigarettes might be associated with some cancers, there is a fundamental difference between smoking and consuming alcoholic beverages: there are NO health benefits to smoking. In contrast, light to moderate wine consumption was shown to have positive effects on cardiovascular and all-cause mortality $[15,17,18]$. Total mortality as endpoint would be a better indicator to assess the risk.

5. This study does not provide any evidence that contradicts the J-shaped relationship between drinking and all-cause mortality $[12,19]$.

6. In addition and most importantly, it does not take into account any lifestyle factors such as diet, body weight, physical activity, family history that may influence the risk of cancer. For beneficial health effects of wine, 
the pattern of drinking is especially important: i.e. consumimg light to moderate amounts regularly, and preferably with food $[6,12,20]$.

Despite these limitations, the authors claim that there is now robust evidence that low levels of alcohol intake do not provide any protective health benefits by referring to the data of the GBD study. However, decades of numerous epidemiological studies backed up by biological experiments do not support such statement. The findings that moderate wine/alcohol consumption reduces the risk of death from heart disease by $25 \%$ [21] and total mortality [19] are omitted.

In contrast, there is a slim body of epidemiological evidence which suggests that moderate intake of alcoholic beverages might have a small effect on breast cancer $[14,20,22]$.

Light drinking, defined as up to 11 units per week, ie. a bottle of wine, is associated with lower cancer rates in some sites and is not associated with breast cancer mortality. Only when consuming 11 to 22 drinks per week, there is an increased mortality risk from any form of cancer ( $+4 \%$ breast cancer) [22].

The claim that there is no safe level of drinking does not seem justifiable with the current scientific evidence.

A handful studies indicate a small relationship between light/moderate drinking and a few cancers while dismissing a much larger body of evidence showing a strong, large and clinically relevant protective association between light/moderate drinking and cardiovascular disease. The protective effect of alcohol on the most important health outcome, total mortality, cannot be ignored. The fact remains that the positive effects of moderate drinking on cardiovascular disease and other conditions exceed and outweigh the negative effects on cancer risk [23]. To focus on the risks while ignoring the benefits is not objective evidence-based science.

Unfortunately, studies such as "How many cigarettes fit into a bottle of wine?" do not contribute to educating consumers about a responsible drinking behaviour to make informed decisions but instead create uncertainty.

\section{Conclusion}

Headlines can be very confusing, thus, it is very important to critically analyse the scientific publications and put the findings into perspective. Population-based studies (such as the GBD study) cannot be interpreted at an individual level. In particular, health professionals who act as multipliers of the public health message/responsible drinking message need to be educated, so they can inform their patients about the individual risk. Family history, cultural environment but also lifestyle factors such as diet, drinking patterns (with the meal in a non-binge drinking way), type of alcoholic beverage, physical exercise, etc. should be part of the individual risk assessment.

According to the current scientific evidence, low to moderate wine consumption with the meals (in a non binge-drinking pattern) has more health benefits than risks and can be part of a health lifestyle.

\section{References}

[1] A.M. Wood, S. Kaptoge, A.S. Butterworth, Lancet 391, 1513 (2018)

[2] E. Gakidou, Gobal Burden of Disease 2016 Alcohol Collaborators Study 1990-2016, Lancet 31310 (2018)

[3] Y. Li, A. Pan, D.D. Wang, X. Liu, K. Dhana, O.H. Franco, S. Kaptoge, E. Di Angelantonio, M. Stampfer, W.C. Willett, F.B. Hu, Circulation 137 (2018)

[4] J. Rehm, R. Room, Nordic Studies on Alcohol and Drugs 34, 330 (2017)

[5] X. Wang, X. Yang, J. Li, et al, Cancer (2019)

[6] A. Giacosa, R. Barale, L. Bavaresco, M.A. Faliva, V. Gerbi, C. La Vecchia, E. Negri, A. Opizzi, S. Perna, Pezzotti, M. Rondanelli, Crit. Rev. Food Sci. Nutr. 56, 635 (2016)

[7] S. Costanzo, A. di Castelnuovo M.B. Donati, L. Iacoviello, G. de Gaetano, Eur. J. Epidemiol. 26, 833 (2011)

[8] M. Gronbaek, U. Becker, D. Johansen, A. Gottschau, P. Schnohr, H.O. Hein, G. Jensen, T.I. Sorensen, Ann. Intern. Med. 133, 411 (2000)

[9] M. Boban, C. Stockley, P.L. Teissedre, P. Restani, U. Fradera, C. Stein-Hammer, J.C. Ruf, Food Funct. 7, 2937 (2016)

[10] A. Gea, M. Bes-Rastrollo, E. Toledo, M. GarciaLopez, J.J. Beunza, R. Estruch, M.A. MartinezGonzalez, Br. J. Nutr. 11, 1871 (2014)

[11] C. Probst, M. Roerecke, S. Behrendt, J. Rehm, Int. J. Epidemiol. 43, 1314 (2014)

[12] D. Eleftheriou, V. Benetou, A. Trichopoulou, C. La Vecchia, C. Bamia, Br. J. Nutr. 120, 1081 (2018)

[13] K. Perreault, A. Bauman, N. Johnson N, Br. J. Sports Med. 51, 651 (2017)

[14] A.L. Klatsky, N. Udaltsova, Y. Li, D. Baer, N.H. Tran, G.D. Friedman, Cancer Causes Control 25, $693(2014)$

[15] J.H. O'Keefe, S.K. Bhatti, A. Bajwa, J.J. Dinicolantonio, C.J. Lavie, Mayo Clin. Proc. 89, 382 (2014)

[16] T.J. Hydes, R. Burton, H. Inskip, M.A. Bellis, N. Sheron, BMC Public Health 19, 316 (2019)

[17] V. Colpani, C.P. Baena, L. Jaspers, G.M. van Dijk, Z. Farajzadegan, K. Dhana, M.J. Tielemans, T. Voortman, R. Freak-Poli, G.G.V. Veloso, R. Chowdhury, M. Kavousi, T. Muka, O.H. Franco, Eur. J. Epidemiol. (2018)

[18] E. Fragopoulou, M. Choleva, S. Antonopoulou, C.A. Demopoulos, Metabolism (2018)

[19] B. Xi, S.P. Veeranki, M. Zhao, C. Ma, Y. Yan, J. Mi, J. Am. Coll. Cardiol. 70, 913 (2017)

[20] L. Schwingshackl, C. Schwedhelm, C. Galbete, G. Hoffmann, Nutrients 9, E1063 (2017)

[21] P.E. Ronksley, S.E. Brien, B.J. Turner, A.K. Mukamal, W.A. Ghali, BMJ. 342, d671 (2011)

[22] Y.J. Choi, S.K. Myung, J.H. Lee, Cancer Res. Treat. 50, 474 (2017)

[23] A.T. Kunzmann, H.G. Coleman, W. Huang, S.I. Berndt, PLOS Med. 15, e1002585 (2018) 